\title{
Including lateral interactions into microkinetic models of catalytic reactions
}

\author{
Hellman, Anders; Honkala, Johanna Karoliina
}

Published in:

Journal of Chemical Physics

Link to article, DOI:

$10.1063 / 1.2790885$

Publication date:

2007

Document Version

Publisher's PDF, also known as Version of record

Link back to DTU Orbit

Citation (APA):

Hellman, A., \& Honkala, J. K. (2007). Including lateral interactions into microkinetic models of catalytic reactions. Journal of Chemical Physics, 127(19), 194704. https://doi.org/10.1063/1.2790885

\section{General rights}

Copyright and moral rights for the publications made accessible in the public portal are retained by the authors and/or other copyright owners and it is a condition of accessing publications that users recognise and abide by the legal requirements associated with these rights.

- Users may download and print one copy of any publication from the public portal for the purpose of private study or research.

- You may not further distribute the material or use it for any profit-making activity or commercial gain

- You may freely distribute the URL identifying the publication in the public portal

If you believe that this document breaches copyright please contact us providing details, and we will remove access to the work immediately and investigate your claim. 


\title{
Including lateral interactions into microkinetic models of catalytic reactions
}

\author{
A. Hellman ${ }^{\text {a) }}$ \\ Haldor Topsфe A/S, Nymфllevej 55, DK-2800 Lyngby, Denmark \\ K. Honkala ${ }^{\text {b) }}$ \\ Center for Atomic-scale Materials Design, Department of Physics, nanoDTU, Technical University \\ of Denmark, DK-2800 Lyngby, Denmark
}

(Received 22 March 2007; accepted 5 September 2007; published online 16 November 2007)

In many catalytic reactions lateral interactions between adsorbates are believed to have a strong influence on the reaction rates. We apply a microkinetic model to explore the effect of lateral interactions and how to efficiently take them into account in a simple catalytic reaction. Three different approximations are investigated: site, mean-field, and quasichemical approximations. The obtained results are compared to accurate Monte Carlo numbers. In the end, we apply the approximations to a real catalytic reaction, namely, ammonia synthesis. () 2007 American Institute of Physics. [DOI: 10.1063/1.2790885]

\section{INTRODUCTION}

Microkinetic models ${ }^{1,2}$ are an essential tool to develop an atomistic understanding of catalysis. They have been successfully applied to various industrially relevant catalytic reactions, such as methanol synthesis ${ }^{3}$ and decomposition, ${ }^{4,5}$ water-gas shift, ${ }^{6}$ ethylene oxidation, ${ }^{7,8}$ and ammonia synthesis. ${ }^{9-11}$ These examples show that the predictive power of microkinetic models can bridge over the temperature, pressure, and material gaps, which exist between fundamental surface science studies and industrially relevant catalysis.

The starting point for constructing a microkinetic model is a proposed reaction mechanism based on our atomistic knowledge of a certain catalytic reaction. The reaction mechanism is then used to write down an explicit expression for a catalytic rate per active site. For a more detailed description of an active site we refer to Fig. 1 and later discussion in the text. Usually it is necessary to make some approximations to get a solvable model. The most common approximation is the assumption of an ideal surface layer, where lateral interactions are excluded. They are, however, well known to often have a dramatic influence on the kinetics of heterogeneous catalytic reactions (see, e.g., reviews in Refs. 12 and 13 and references therein). Nowadays lateral interactions between reaction intermediates can be calculated using density functional theory ${ }^{10,14,15}$ and then included into microkinetic models by means of Monte Carlo (MC) techniques. ${ }^{10}$ For a review of MC simulations of catalytic reactions, see Refs. 13, 16, and 17.

We distinguish between two types of lateral interactions: one for adsorbate-adsorbate interactions in an adsorbed state and one for adsorbate-transition state interactions in an activated state. By an adsorbed state we mean an adsorbate sitting at an optimized adsorption site, whereas an activated

\footnotetext{
${ }^{\text {a) }}$ Present address: Competence Centre for Catalysis Chalmers University of Technology SE-412 96 Göteborg, Sweden. Electronic mail: ahell@fy.chalmers.se

${ }^{b}$ Present address. Department of Chemistry, Nanoscience Center, P.O.Box 35, FIN-40014 University of Jyväskylä, Finland.
}

state refers to a reaction intermediate at the transition state. The lateral interactions of the adsorbed state have a twofold influence on the local adsorbate configuration at the active site; (i) they change the coverage of different adsorbates and (ii) they introduce correlation among adsorbates. At the activated state lateral interactions depend on the local adsorbate configuration at the active site and modify the transition state energy accordingly.

In this paper, three different approximations to treat lateral interactions are applied. The most simple one is the site approximation $^{12,18}(\mathrm{SA})$, where the lateral interactions are completely ignored. In the mean-field (MF) approximation ${ }^{12,18}$ average lateral interactions are used, and they depend on the coverage of different adsorbates. The quasichemical approximation ${ }^{12,18,19}(\mathrm{QCA})$ is the most accurate one, and it includes the explicit treatment of lateral interactions between two adjacent sites. In addition, three different surface geometries are considered, (i) one-dimensional a)

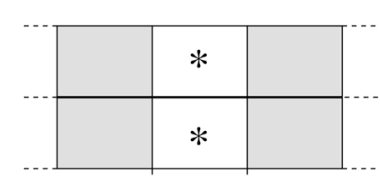

c)

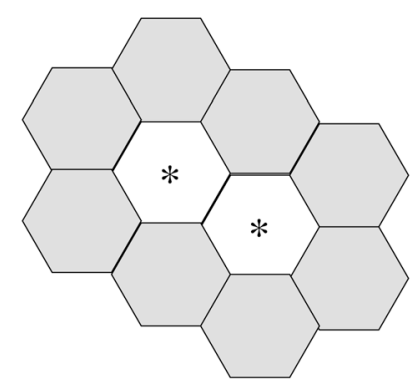

b)

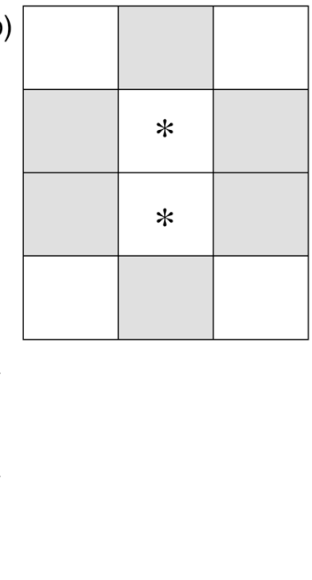

FIG. 1. Schematic figure of the three active sites that are considered. Star $(\star)$ indicates an empty site, whereas a neighboring site is indicated in gray. (a) In the one-dimensional surface lattice the active sites consist of $3+3$ surface sites. (b) In the square lattice the active sites consist of eight surface sites. (c) In the sixfold lattice the active sites consist of ten surface sites. 
(1D) lattice to simulate reactions that occur at surface steps, (ii) a square lattice, and finally (iii) a sixfold lattice. In the end the catalytic rates calculated using the above approximations on each surface geometry are compared to accurate MC simulations that fully incorporate the nearest neighbor lateral interactions. It should be noted that our interaction model is simple and we ignore higher order interactions, such as three-body interactions and long-ranged interactions. However, within a defined interaction model the MC results are accurate.

\section{THE MICROKINETIC MODEL}

To study the accuracy of the approximations, we use a simple surface reaction, $A_{2}+B_{2} \rightleftharpoons 2 A B$, which is assumed to follow a Langmuir-Hinshelwood reaction path, where each reaction step obeys microscopic reversibility,

$$
\begin{aligned}
A_{2}+2 * & \rightleftharpoons 2 A *, \\
B_{2} *+2 * & \rightleftharpoons 2 B *, \\
A *+B * & \rightleftharpoons A B *+*, \\
A B * & \rightleftharpoons A B+* .
\end{aligned}
$$

Here $*$ and $x *$ correspond to an empty site and an adsorbate $x=A, B, A B$, respectively. Although the reaction is simple, it includes most of the intrinsic features involved in a real catalytic reaction: the activation (dissociation) of the reactants, followed by the removal of dissociation products by surface reactions, and then finally the removal of the products from the surface (desorption). Several important catalytic reactions can be described in such a phenomenological fashion, ${ }^{20}$ for instance, ammonia synthesis, Fischer-Tropsch synthesis, NO reduction, and many oxidation reactions.

To keep the microkinetic model traceable, we assume that the catalytic reaction contains a rate-limiting step for which we, in this case, choose $A_{2}$ dissociation. We also assume that all other reaction steps are in quasiequilibrium. Hence, the rate per active site is

$$
r=p_{A_{2}} \nu \sum_{i} \mathbf{P}_{i} \exp \left(-E_{i}^{a} / k_{B} T\right),
$$

where $p_{A_{2}}$ is the partial pressure of $A_{2}, \nu$ is the prefactor for $A_{2}$ dissociation, $\mathbf{P}_{i}$ is the probability of finding a local adsorbate configuration $i$ at the active site, and $E_{i}^{a}$ is the activation energy for $A_{2}$ dissociation. To investigate the effect of lateral interactions at the activated state, $E_{i}^{a}$ is required to depend on the local adsorbate configuration $i$ at the active site. This is included by assuming that every occupied nearest neighbor site increases $E_{i}^{a}$ by the same amount. In this model study the activation energy $E_{i}^{a}$ is chosen to be $30 \mathrm{~kJ} / \mathrm{mol}$ on a clean surface, whereas each nearest neighbor adsorbate adds $10 \mathrm{~kJ} / \mathrm{mol}$ to $E_{i}^{a}$. These values, although reasonable, are of no significant importance to the conclusions, as they only affect the absolute magnitude of the rates and our conclusions are based on normalized rates.

We use three different surface lattices to model the ac-

\begin{tabular}{|c|c|c|c|c|}
\hline $\begin{array}{c}\mathrm{US}_{\mathrm{H}-\mathrm{H}} \\
E_{\mathrm{int}}=0.024\end{array}$ & $\begin{array}{c}\mathrm{US}_{\mathrm{H}-\mathrm{N}} \\
E_{\mathrm{int}}=0.13\end{array}$ & $\begin{array}{c}\mathrm{US}_{\mathrm{H}-\mathrm{NH}} \\
E_{\mathrm{int}}=0.075\end{array}$ & $\begin{array}{c}\mathrm{US}_{\mathrm{H}-\mathrm{NH}} \\
E_{\mathrm{int}}=-0.05\end{array}$ & $\begin{array}{l}\mathrm{US}_{\mathrm{H}-\mathrm{NH}_{3}} \\
E_{\text {int }}=0.0\end{array}$ \\
\hline $\begin{array}{c}\mathrm{US}_{\mathrm{N}-\mathrm{N}} \\
E_{\mathrm{int}}=0.38\end{array}$ & $\begin{array}{c}\mathrm{US}_{\mathrm{N}-\mathrm{NH}} \\
E_{\mathrm{int}}=0.24\end{array}$ & $\begin{array}{l}\mathrm{US}_{\mathrm{N}-\mathrm{NH}_{2}} \\
E_{\mathrm{int}}=0.12\end{array}$ & $\begin{array}{c}\mathrm{US}_{\mathrm{N}-\mathrm{NH}_{3}} \\
E_{\text {int }}=-0.16\end{array}$ & $\begin{array}{l}\mathrm{US}_{\mathrm{NH}-\mathrm{NH}} \\
E_{\mathrm{int}}=0.21\end{array}$ \\
\hline $\begin{array}{l}\mathrm{US}_{\mathrm{NH}-\mathrm{NH}_{2}} \\
E_{\mathrm{int}}=0.25\end{array}$ & $\begin{array}{l}\mathrm{US}_{\mathrm{NH}-\mathrm{NH}_{3}} \\
E_{\text {int }}=0.18\end{array}$ & $\begin{array}{c}\mathrm{US}_{\mathrm{NH}_{2}-\mathrm{NH}_{2}} \\
E_{\mathrm{int}}=0.43\end{array}$ & $\begin{array}{c}\mathrm{US}_{\mathrm{NH}_{2}-\mathrm{NH}_{3}} \\
E_{\mathrm{int}}=0.30\end{array}$ & $\begin{array}{c}\mathrm{US}_{\mathrm{NH}_{2}-\mathrm{NH}_{3}} \\
E_{\text {int }}=0.16\end{array}$ \\
\hline $\begin{array}{c}\mathrm{LS}_{\mathrm{H}-\mathrm{H}} \\
E_{\mathrm{int}}=0.06\end{array}$ & $\begin{array}{c}\mathrm{LS}_{\mathrm{H}-\mathrm{N}} \\
E_{\mathrm{int}}=0.16\end{array}$ & $\begin{array}{c}\mathrm{LS}_{\mathrm{H}-\mathrm{NH}} \\
E_{\mathrm{int}}=0.14\end{array}$ & $\begin{array}{c}\mathrm{LS}_{\mathrm{H}-\mathrm{NH}} \\
E_{\mathrm{int}}=0.05\end{array}$ & $\begin{array}{c}\mathrm{LS}_{\mathrm{H}-\mathrm{NH}_{3}} \\
E_{\text {int }}=0.12\end{array}$ \\
\hline $\begin{array}{c}\mathrm{LS}_{\mathrm{N}-\mathrm{N}} \\
E_{\mathrm{int}}=0.35\end{array}$ & $\begin{array}{c}\mathrm{LS}_{\mathrm{N}-\mathrm{NH}} \\
E_{\mathrm{int}}=0.27\end{array}$ & $\begin{array}{c}\mathrm{LS}_{\mathrm{N}-\mathrm{NH}_{2}} \\
E_{\mathrm{int}}=0.21\end{array}$ & $\begin{array}{c}\mathrm{LS}_{\mathrm{N}-\mathrm{NH}_{3}} \\
E_{\text {int }}=-0.02\end{array}$ & $\begin{array}{l}\mathrm{LS}_{\mathrm{NH}-\mathrm{NH}} \\
E_{\mathrm{int}}=0.27\end{array}$ \\
\hline $\begin{array}{l}\mathrm{LS}_{\mathrm{NH}-\mathrm{NH}_{2}} \\
E_{\mathrm{int}}=0.29\end{array}$ & $\begin{array}{l}\mathrm{LS}_{\mathrm{NH}-\mathrm{NH}_{3}} \\
E_{\mathrm{int}}=0.08\end{array}$ & $\begin{array}{c}\mathrm{LS}_{\mathrm{NH}_{2}-\mathrm{NH}_{2}} \\
E_{\mathrm{int}}=0.30\end{array}$ & $\begin{array}{c}\mathrm{LS}_{\mathrm{NH}_{2}-\mathrm{NH}_{3}} \\
E_{\mathrm{int}}=0.30\end{array}$ & $\begin{array}{c}\mathrm{LS}_{\mathrm{NH}_{3}-\mathrm{NH}_{3}} \\
E_{\text {int }}=0.59\end{array}$ \\
\hline
\end{tabular}
tive site where the catalytic reaction occurs, see Fig. 1. Note
TABLE I. Lateral interaction energies relevant for ammonia synthesis. US and LS stand for upper and lower steps, respectively. For further details, see Ref. 10. Energies are in eV.

that the active site consists of several surface sites. The simplest lattice model is one dimensional and represents an active site at a surface step. The active site consists of an upper step (US) and a lower step (LS), see Fig. 1, which are independent of each other, that is, there are no lateral interactions between US and LS. This implies that each surface site has two neighbors. Note that in the simplified microkinetic model presented here, the interactions on the upper and lower steps are considered to be equal for simplicity. However, for ammonia synthesis we use interaction parameters given in Table I and they differ for US and LS. The second lattice model is a two-dimensional (2D) square lattice, where each surface site has four neighbors. The last lattice model is a sixfold lattice, where each surface site has six neighbors. As the $A_{2}$ dissociation requires two adjacent empty sites, the local adsorbate configuration $i$ at the active site is determined by the occupancy of the neighboring surface sites surrounding these empty surface sites, that is, (i) in the onedimensional case two neighboring sites on the US and two neighboring sites on the LS, (ii) in the square lattice case six neighboring sites, and finally (iii) in the sixfold lattice eight neighboring sites, see Fig. 1.

Finally, there is a question of how to calculate $\mathbf{P}_{i}$ in Eq. (5). The probability can be directly determined with MC techniques or by solving the algebraic equilibrium equations derived within the microkinetic model.

As reaction steps [Eqs. (2)-(4)] are considered to be in quasiequilibrium, the coverages of the different adsorbates are

$$
\begin{aligned}
& \theta_{*}=\frac{1}{1+\frac{p_{A B}}{\sqrt{p_{B_{2}} K_{2}} K_{3} K_{4}}+\sqrt{p_{B_{2}} K_{2}}+\frac{p_{A B}}{K_{4}}}, \\
& \theta_{A}=\frac{p_{A B}}{\sqrt{p_{B_{2}} K_{2} K_{3} K_{4}} \theta_{*},} \\
& \theta_{B}=\sqrt{p_{B_{2}} K_{2}} \theta_{*},
\end{aligned}
$$




$$
\theta_{A B}=\frac{p_{A B}}{K_{4}} \theta_{*},
$$

where equilibrium constants are

$$
\begin{aligned}
& K_{2}=\frac{z_{B_{*}}^{2}}{z_{B_{2}}} \exp \left[-\beta 2 \bar{E}_{B^{*}}\right], \\
& K_{3}=\frac{z_{A B_{*}}}{z_{A_{*} z_{B_{*}}}} \exp \left[-\beta\left(\bar{E}_{A B_{*}}-\bar{E}_{A_{*}}-\bar{E}_{B_{*}}\right)\right], \\
& K_{4}=\frac{z_{A B}}{z_{A B_{*}}} \exp \left[\beta \bar{E}_{A B_{*}}\right] .
\end{aligned}
$$

Here, $\beta=1 / k_{B} T$ and the partition functions $z_{x}, x=A, B, A B$ include vibrational and rotational informations of the adsorbates. We assume that the partition functions are independent of lateral interactions. The parameters used in the model are as follows: the free energy of adsorbate $A, B, A B$ is chosen so that at $T=500 \mathrm{~K}$ the coverage of each adsorbate is $1 / 4 \mathrm{ML}$ without lateral interactions. Although this is a reasonable choice, we emphasize that it does not affect our conclusions. The solution to the rate equation depends only on the effective adsorption energies of the different adsorbates, $\bar{E}_{A_{*}}, \bar{E}_{B_{*}}, \bar{E}_{A B_{*}}$, which in turn depend on the lateral interactions between adsorbates. In this study we consider only lateral interactions between nearest neighbor sites.

\section{Site approximation}

In the $\mathrm{SA},{ }^{12,18}$ all sites are independent of their surroundings, thus lateral interactions are excluded. This means that the nearest neighbor adsorbates do not contribute to the effective adsorption energy, i.e.,

$$
\bar{E}_{x}=E_{x},
$$

where $E_{x}$ is the standard adsorption energy of adsorbate $x$.

As the SA ignores lateral interactions, the occupancy of adjacent sites is not correlated. The probability $\mathbf{P}_{i}$ of a certain local adsorbate configuration at the active site is equal to

$$
\mathbf{P}_{i}=c \prod_{n=1}^{N} \theta_{n, x} \theta_{*}^{2},
$$

where on the considered surface lattice $c$ is the number of possible ways to find two adjacent sites that are empty, $\theta_{n, x}$ is the coverage of adsorbate $x$ at the neighboring site $n$, and $N$ is the number of neighboring sites.

\section{Mean-field approximation}

In the MF (Refs. 12 and 18) approximation average lateral interactions are taken into account. More explicitly, the effective adsorption energy is adjusted to the lateral interactions between the considered adsorbate and the coverage of all adsorbates according to

$$
\bar{E}_{x}=E_{x}+N \sum_{\dot{x}} \theta_{\dot{x}} w_{x-\dot{x}},
$$

where $w_{x-\dot{x}}$ is the interaction between adsorbates $x$ and $\dot{x}$. Note that the summation over the number of neighboring sites is accounted for in Eq. (15) by including $N$.

One consequence of Eq. (15) is that the effect of repulsive/attractive short range interactions, like the nearest neighbor lateral interactions used here, will be overestimated/underestimated. This is an intrinsic feature of the MF approximation since adsorbate-adsorbate correlation, which could reduce/increase the effect of nearest neighbor lateral interactions, is not taken into account. For the same reason, the probability $\mathbf{P}_{i}$ of a certain local adsorbate configuration at the active site is same as in Eq. (14).

\section{Quasichemical approximation}

The QCA (Refs. 12, 18, and 19) includes the correlation between two surface sites. Writing up the grand-canonical distribution for the two surface sites, and taking explicitly the lateral interactions between different adsorbates into account, the following relationship between adsorbate pair probabilities can be derived:

$$
\frac{P_{x x} P_{\dot{x} \dot{x}}}{P_{x \dot{x}}^{2}}=\exp \left[-\beta\left(2 w_{x-\dot{x}}-w_{x-x}-w_{\dot{x}-\dot{x}}\right)\right],
$$

where $P_{x x}, P_{\dot{x} x}, P_{x \dot{x}}$ are the probabilities that two neighboring sites are occupied by $x x, \dot{x} \dot{x}, x \dot{x}$ adsorbate pairs. Here we use $P_{x \dot{x}}=P_{\dot{x} x}$. Both $x$ and $\dot{x}$ are summed over all possible adsorbates.

In addition, we have two balance equations,

$$
\begin{aligned}
& \sum_{x \dot{x}} P_{x \dot{x}}=1, \\
& \sum_{\dot{x}} P_{x \dot{x}}=\theta_{x} .
\end{aligned}
$$

In the case of a single adsorbate, e.g., $x=A$, these equations have an analytic solution. ${ }^{12}$ However, for larger number of adsorbates the equations must be solved numerically. For instance, if $\Sigma_{x} P_{x x}^{(m)}$ is the joint probability at step $m$, an iterative solution ${ }^{12}$ is given,

$$
\begin{aligned}
& P_{x \dot{x}}^{(m+1)}=\sqrt{\frac{P_{x x}^{m} P_{\dot{x} \dot{x}}^{m}}{\exp \beta\left(2 w_{x-\dot{x}}-w_{x-x}-w_{\dot{x}-\dot{x}}\right)}}, \\
& P_{x x}^{(m+2)}=P_{x x}^{m} \theta_{x} /\left(P_{x x}^{m}+\sum_{\dot{x} \neq x} P_{x \dot{x}}^{(m+1)}\right),
\end{aligned}
$$

where $m$ is increased until a stable solution is reached.

The effective adsorption energy is determined by

$$
\bar{E}_{x}=E_{x}+\frac{N}{\beta} \ln \left[\sum_{\dot{x}} P\left(\theta_{x} \mid \theta_{\dot{x}}\right) \exp \left(\beta w_{x-\dot{x}}\right)\right],
$$

where $P\left(\theta_{x} \mid \theta_{\dot{x}}\right)$ is the conditional probability that adsorbate $x$ has $\dot{x}$ as a nearest neighbor. This expression includes both the energy of lateral interactions and the correlation between the different adsorbates. 
As the QCA includes the correlation due to lateral interactions, the probability $\mathbf{P}_{i}$ of a certain local adsorbate configuration at the active sites is equal to

$$
\mathbf{P}_{i}=c \prod_{n=1}^{N} P\left(\theta_{n, x} \mid \theta_{*}\right) \theta_{*} P\left(\theta_{*} \mid \theta_{*}\right),
$$

where for simplicity the probability of finding two adjacent sites empty equals to $\theta_{*} P\left(\theta_{*} \mid \theta_{*}\right)$ and $c$ is the number of possible permutations on the considered surface lattice of these two adjacent empty sites. $P\left(\theta_{n, x} \mid \theta_{*}\right)$ stands for the conditional probability of having one specific neighbor $x$ at site $n$ next to an empty site and $N$ is the total number of neighboring sites.

\section{Monte Carlo}

The free energies and lateral interactions of all reaction intermediates together with gas-phase reservoir of $B_{2}$ and $A B$ completely determine the thermodynamical equilibrium of the studied system represented by a grand-canonical ensemble. The thermodynamic equilibrium of the ensemble is obtained by means of MC techniques, ${ }^{13,21}$ where the configuration space is sampled according to the METROPOLIS algorithm. The Monte Carlo technique is used to make sure that the surfaces coverages and adsorbate-adsorbate correlations are calculated in the correct way. The equilibrium condition includes association/dissociation reactions and adsorption/ desorption events. The transition probability between various state configurations satisfies the detail balance principle. The gas in the reservoir is described by pressure- and temperature-dependent chemical potentials ${ }^{18}$ for $B_{2}$ and $A B$,

$$
\mu_{B}\left(T, p_{B_{2}}\right)=\frac{1}{2 \beta} \ln \frac{p_{B_{2}}}{p_{0} z_{B_{2}}}
$$

and

$$
\mu_{A B}\left(T, p_{A B}\right)=\frac{1}{\beta} \ln \frac{p_{A B}}{p_{0} z_{A B}},
$$

where the reference pressure $p_{0}$ is 1 bar.

From the thermodynamic equilibrium any relevant statistical information can be extracted (coverage and conditional probability) by analyzing the ensemble of configurations obtained within the MC simulation. ${ }^{22}$ More specifically, the coverage $\theta_{x}$ is determined by calculating the occurrence of a certain adsorbate $x$, whereas the conditional probability is determined from the ensemble of local adsorbate configurations obtained in the MC simulation. The probability $\mathbf{P}_{i}$ of a certain local adsorbate configuration at the active sites is calculated according Eq. (22). For more details on ammonia synthesis, please see Ref. 10.

\section{RESULTS AND DISCUSSION}

To visualize the effect of lateral interactions on the rate, we introduce a phenomenological interaction parameter defined as
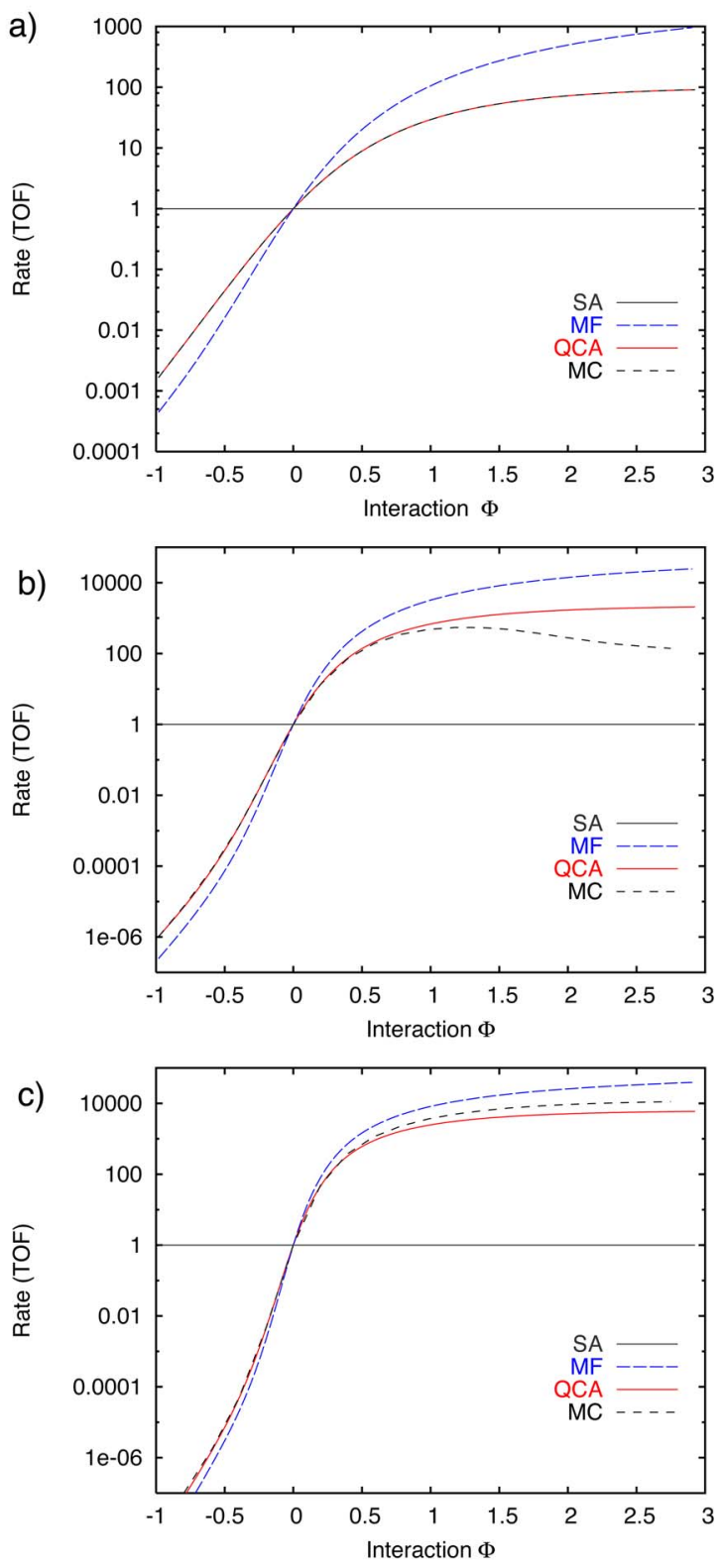

FIG. 2. (Color online) The calculated normalized rate as a function of the phenomenological interaction parameter for a catalytic reaction taking place on (a) 1D step, (b) square lattice, and finally (c) sixfold lattice.

$$
\Phi=\sum_{x \dot{x}} \frac{w_{x-\dot{x}}}{F_{x}}
$$

where $F_{x}$ is the free energy of an adsorbate $x$. The mathematical form of $\Phi$ is not important as long as it is kept same during the comparison. The main advantage of Eq. (25) is that it includes the fact that strongly adsorbed adsorbates require also large lateral interactions before their coverages change. We admit that the order parameter is rather crude but it enables us to measure and compare the quality of the three different approximations. The lateral interactions, $w_{x-\dot{x}}$, used in the ground state, range from -5 (attraction) to $15 \mathrm{~kJ} / \mathrm{mol}$ (repulsion). Figures 2(a)-2(c) present calculated rates as a function of the interaction parameter $\Phi$ for the three different interaction approximations and surface lattices. To keep the results traceable, the lateral interactions are increased simul- 
taneously by the same amount and all the results are normalized to the rate obtained in the SA.

Figure 2(a) shows the reaction rate at the 1D lattice for different approximations as well as for MC simulations. The rate depends rather weakly on the interaction parameter, which is due to the low number of nearest neighbor sites. Note that at attractive interactions, $\Phi<0$, the SA overestimates and MF underestimates the rate, whereas at repulsive interactions, $\Phi>0$, SA (MF) underestimates (overestimates), respectively. For the 1D lattice QCA reproduces the MC results for all $\Phi$ values. The ability of the QCA to give exact results in 1D (Refs. 12, 18, and 23) was, for instance, recently exploited in a study of interacting polyatomic adsorbates. ${ }^{24}$

For the square lattice, the rate is found to be larger/ smaller compared to the 1D case with the same repulsive/ attractive value of the interaction parameter $\Phi$, see Fig. 2(b). The reason for this is the larger number of nearest neighbor sites, which effectively gives rise to larger lateral interactions. The qualitative behavior of the SA and MF approximations with respect to QCA and MC is exactly the same as in the 1D case. However, now also the QCA results differ from the MC ones. The discrepancy arises from the two dimensionality of the lattice as the two sites considered in the QCA can now implicitly affect each other through the other sites that are connected in the lattice, see Fig. 1. This becomes particularly important when lateral interactions increase. Note that the rate becomes smaller at larger values of the interaction parameter, i.e., the adsorbate-adsorbate correlation reduces the probability to find two adjacent empty sites on a square lattice.

Compared to the $1 \mathrm{D}$ or the square lattice cases the rate is found, as expected, to be even larger/smaller for the sixfold lattice with the same repulsive/attractive value of the interaction parameter $\Phi$, see Fig. 2(c). The reason is that the number of nearest neighbor sites is further increased, which gives rise to even larger lateral interactions. Although the quality of the QCA results is far better than those given by the two other approximations, we notice that the QCA deviates from the MC results already at $\Phi=0.5$, which is 0.25 lower than seen for QCA with the square lattice. The difference does not only result from the larger number of nearest neighbor sites but also from the fact that the two surface sites explicitly considered in the QCA now share common nearest neighbor sites. However, the qualitative behavior of the SA and MF approximations with respect to QCA and MC is the same as for the other two lattices.

The above analysis shows that the rates calculated with the SA approximation are unreliable, even at the low interaction parameter values. The two other approximations, MF and QCA, seem to work well at low and intermediate interaction strengths. However, with increasing value of the interaction parameter, the discrepancy between the exact MC results and different approximations becomes more severe. The SA differs by a factor of 100 (1D case) to a factor of 10000 (2D sixfold case) at $\Phi=1.0$, whereas the MF and QCA do much better and differ only by factors of 10-100 (MF) and 1-100 (QCA). The fact that QCA can fully reproduce the $\mathrm{MC}$ results in the 1D case is particularly interesting. Several

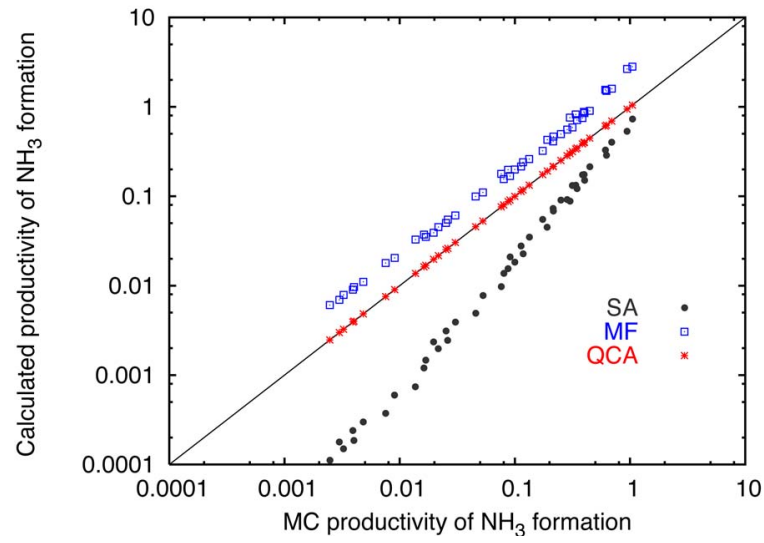

FIG. 3. (Color online) The calculated productivity of ammonia from a theoretical plug-flow reactor depending on the level of approximation. The solid line is based on MC values. Adsorption energies, lateral interactions, and activation energies are all calculated from first principles. For further information, see Refs. 10 and 11.

experimental results ${ }^{25-27}$ show that surface defects, such as steps, are mainly responsible for the activity in many catalytic reactions. The surface defects resemble 1D structures, hence coverages and lateral interactions on these defects can be addressed in an exact manner using the QCA.

\section{A REAL CATALYTIC REACTION}

As a final step we study a real catalytic reaction: ammonia synthesis. A complete first-principles model of the ammonia synthesis over an unpromoted Ru-based catalyst was recently published. ${ }^{10,11}$ In that work, lateral interactions between all reaction intermediates were fully accounted for by means of MC techniques. Here we apply the three different approximations and compare the results to those obtained with MC. As step sites totally dominate the catalytic activity, ${ }^{10,25}$ the $1 \mathrm{D}$ lattice is the most suitable one.

Under the synthesis conditions (total pressure: 50-100 bars, temperature: 593-713 K, flow: $30-200 \mathrm{~N} \mathrm{ml} / \mathrm{min}$, and at stoichiometric $\mathrm{H}_{2}: \mathrm{N}_{2}$ ratio) the most dominate reaction intermediate is adsorbed $\mathrm{H}^{10,11}$ As the $\mathrm{N}_{2}$ dissociation is the rate-determining step, the distribution of adsorbed particles is considered to be canonical and we assume that the diffusion of the adsorbates is fast, at least on the time scale for $\mathrm{N}_{2}$ dissociation. The lateral interaction between adsorbed $\mathrm{H}$ and all other reaction intermediates lies in the intermediate range of interaction strength, $\Phi \sim 1.0$, see Table I and Ref. 10 for details. The SA, MF, and QCA results follow our earlier analysis, that is, SA underestimates by a factor of $10-100$, MF overestimates by a factor of 5-10, and QCA reproduces the real ammonia productivity. However, there is also a quantitative difference in the slope of the productivities depending on which approximation is considered, see Fig. 3. The reason is that the effects arising from lateral interactions depend indirectly on macroscopic parameters, such as temperature and partial pressures, which is reflected in the results. Therefore we stress that for the quantitative studies, it is important to include lateral interactions into the microkinetic models. 
A common practice to determine parameters needed in a microkinetic model is to fit them to measurements, in which case the lateral interaction is implicitly taken into account. Models with fitted parameters usually reproduce experimental data well at least under reaction conditions similar to those used in experiments. Our results show that small changes in pressure and temperature alter affective adsorption energies. This indicates that the applicability of fitted parameters is limited to the fitting conditions. One way to improve the parameters is to evaluated lateral interactions under various coverages, i.e., at different conditions, and then include them into the fitting procedure via a MFA or QCA approximation.

\section{CONCLUSION}

In this work we have shown that lateral interactions between adsorbed species have a strong influence on the reaction rate of a catalytic process. Three different approximations to describe interactions are used: site, mean-field, and quasichemical approximations, and the results are compared to Monte Carlo simulations. The active site, where the catalytic reaction takes place, has been modeled both with $1 \mathrm{D}$ lattice and with 2D square and hexagonal lattices.

Our results show that the site approximation is inadequate for a heterogeneous catalysis reaction already at weak lateral interactions. The mean-field and quasichemical approximations are much better, and the latter one even fully reproduces the Monte Carlo results at the $1 \mathrm{D}$ lattice. The computational effort increases by a factor of 2-3 from the site approximation to QCA. However, compared to MC simulations the approximations provide substantial savings in computer time, which is necessary for the large scale computational screening of various materials and reactions.

\section{ACKNOWLEDGMENTS}

The authors thank the Danish Center for Scientific Computing for computer resources and economic support through EU Project No. HPRN-CT-2002-00170. K.H. thanks also the Academy of Finland for financial support. Valuable discussions with J. K. Nørskov and V. P. Zhdanov are also acknowledged.
${ }^{1}$ The Microkinetics of Heterogeneous Catalysis, edited by J. A. Dumesic, D. F. Rudd, L. M. Aparicio, J. E. Rekoske, and A. A. Trivino (ACS, Washington, DC, 1993).

${ }^{2}$ P. Stoltze, Prog. Surf. Sci. 65, 65 (2000).

${ }^{3}$ T. Askgaard, J. K. Nørskov, C. V. Ovesen, and P. Stoltze, J. Catal. 156, 229 (1995).

${ }^{4}$ J. Greeley and M. Mavrikakis, J. Am. Chem. Soc. 126, 3910 (2004).

${ }^{5}$ S. Kandoi, J. Greeley, M. A. Sanchez-Castillo, S. T. Evans, A. A. Gokhale, J. A. Dumesic, and M. Mavrikakis, Top. Catal. 37, 17 (2006).

${ }^{6}$ C. V. Ovesen, B. S. Clausen, B. S. Hammershøi, G. Steffensen, T. Askgaard, I. Chorkendorff, J. K. Nørskov, P. B. Rasmussen, P. Stoltze, and P. Taylor, J. Catal. 158, 170 (1996).

${ }^{7}$ S. Linic and M. A. Barteau, J. Am. Chem. Soc. 125, 4034 (2003).

${ }^{8}$ C. Stegelmann, N. C. Schiødt, C. T. Campbell, and P. Stoltze, J. Catal. 221, 630 (2004).

${ }^{9}$ P. Stoltze, Phys. Scr. 36, 824 (1987).

${ }^{10}$ K. Honkala, A. Hellman, I. N. Remediakis, Á. Logadóttir, A. Carlsson, S. Dahl, C. H. Christensen, and J. K. Nørskov, Science 307, 555 (2005).

${ }^{11}$ A. Hellman, K. Honkala, I. N. Remediakis, Á. Logadóttir, A. Carlsson, S. Dahl, C. H. Christensen, and J. K. Nørskov, Surf. Sci. 600, 4264 (2006).

${ }^{12}$ V. P. Zhdanov, Elementary Physicochemical Processes on Solid Surfaces (Plenum, New York, 1991).

${ }^{13}$ H. C. Kang and W. H. Weinberg, Chem. Rev. (Washington, D.C.) 95, 667 (1995).

${ }^{14}$ K. Reuter and M. Scheffler, Phys. Rev. B 73, 045433 (2006).

${ }^{15}$ K. Reuter, D. Frenkel, and M. Scheffler, Phys. Rev. Lett. 93, 116105 (2004).

${ }^{16}$ V. P. Zhdanov, Surf. Sci. Rep. 45, 231 (2002).

${ }^{17}$ D. J. Liu and J. W. Evans, Multiscale Model. Simul. 4, 424 (2005).

${ }^{18}$ T. L. Hill, An introduction to statistical thermodynamics (Dover, New York, 1960)

${ }^{19}$ V. P. Zhdanov, Surf. Sci. 111, 63 (1981)

${ }^{20}$ T. Bligaard, J. K. Nørskov, S. Dahl, J. Matthiesen, and C. H. Christensen, J. Catal. 224, 206 (2004)

${ }^{21}$ D. P. Landau and K. Binder, A Guide to Monte Carlo Simulations in Statistical Physics (Cambridge University Press, Cambridge, 2000).

${ }^{22}$ The lattice consist of 400 sites for the one-dimensional step, $20 \times 20$ sites for the square and sixfold lattice. $4 \times 10^{6} \mathrm{MC}$ steps are simulated and the lattice configuration is sampled at every $4 \times 10^{3} \mathrm{MC}$ step.

${ }^{23}$ A. J. Ramirez-Pastor, T. P. Eggarter, V. D. Pereyra, and J. L. Riccardo, Phys. Rev. B 59, 11027 (1999).

${ }^{24}$ M. Dávila, F. Romá, J. L. Riccardo, and A. J. Ramirez-Pastor, Surf. Sci. 600, 2011 (2006)

${ }^{25}$ S. Dahl, Á. Logadóttir, R. C. Egeberg, J. H. Larsen, E. Törnqvist, I. Chorkendorff, and J. K. Nørskov, Phys. Rev. Lett. 83, 1814 (1999).

${ }^{26}$ S. Helveg, C. Lopez-Cartes, J. Sehested, P. L. Hansen, B. S. Clausen, J. R. Rostrup-Nielsen, F. Abild-Pedersen, and J. K. Nørskov, Nature (London) 427, 426 (2004).

${ }^{27}$ R. T. Vang, K. Honkala, S. Dahl, E. K. Vestergaard, J. Schnadt, E. Lægsgaard, B. S. Clausen, J. K. Nørskov, and F. Besenbacher, Nat. Mater. 4, 160 (2005). 